\title{
Novel genetic variants link ankylosing spondylitis and Crohn disease: evidence of a shared pathogenesis?
}

Results from a genome-wide association study published in PLoS Genetics demonstrate that several susceptibility genes for Crohn disease are also associated with ankylosing spondylitis (AS). "These associations were present even amongst ankylosing spondylitis cases lacking clinically evident Crohn disease," points out Matthew Brown of the University of Queensland, Australia, and the corresponding author of the study.

AS and inflammatory bowel disease (IBD; Crohn disease and ulcerative colitis) often occur in the same families and individuals. Approximately $10 \%$ of patients with AS present with overt IBD, and $70 \%$ have subclinical inflammation in the terminal ileum.

As many more genetic associations have been found for Crohn disease than AS, the researchers genotyped 2,773 unrelated individuals of European ancestry with AS for 53 markers within
30 genetic loci previously linked to Crohn disease. The results were compared with archived genotype data for 2,215 population-matched controls. The known association of $I L 23 R$ with AS was confirmed, and several new loci also correlated strongly with this disease. The most robust association involved a marker close to KIF21B on chromosome 1q32. Polymorphisms within and near STAT3, IL12B, CDKAL1 and LRRK2 or MUC19, as well as an intergenic region on chromosome 13q14, were also associated with AS. Exclusion of patients with overt IBD from the analysis did not weaken these associations.

Interestingly, the proteins encoded by STAT3, IL12B and IL23R are required for production of interleukin (IL)-17 by type $17 \mathrm{~T}$ helper cells, which highlights the involvement of these lymphocytes in the pathogenesis of both AS and Crohn disease.
"Given these findings, and the fact that blockade of IL-12 and IL-23 signaling with ustekinumab is effective in the treatment of Crohn disease, we have been encouraging companies with agents targeting this pathway to study the effect of those agents in ankylosing spondylitis," says Brown. "Our findings indicate that shared genetic susceptibility is a common feature of epidemiologically related diseases," he continues, "and support the use of strategies searching for pleiotropic genetic effects, such as the Immunochip program."

Shreeya Nanda

Original article Danoy, P. et al. Association of variants
at 1q32 and STAT3 with ankylosing spondylitis suggests
genetic overlap with Crohn's disease. PLoS Genet. 6,
e1001195 (2010)
Further reading Elewaut, D. Linking Crohn's disease and
ankylosing spondylitis: it's all about genes! PLoS Genet. 6,
e1001223 (2010)

